\section{SAT0570 \\ RESTING-STATE FMRI BRAIN CONNECTIVITY IN HAND OSTEOARTHRITIS}

M.D. Russell ${ }^{1}$, F.A. Howe ${ }^{2}$, T.R. Barrick ${ }^{2}$, N. Sofat ${ }^{1} .{ }^{1}$ Institute of Infection and Immunity; ${ }^{2}$ Molecular and Clinical Sciences Research Institute, St George's, University of London, London, UK

Background: Studies indicate brain grey matter volumetric changes are associated with chronic pain. In people with painful hand osteoarthritis (OA), grey matter volume reductions have been identified in the anterior cingulate cortex (ACC): a key pain-processing region. ${ }^{1}$

Objectives: We hypothesised that people with hand OA would have alterations in resting-state functional connectivity networks involving the ACC and other painprocessing brain regions relative to non-OA controls. Furthermore, we hypothesised that treatment with centrally-acting analgesics (pregabalin or duloxetine) would result in connectivity changes in these brain regions.

Methods: Resting-state functional MRI (fMRI) of the brain was performed on hand OA participants $(n=28)$ before and after 12 weeks of treatment with duloxetine, pregabalin or placebo, and compared to non-OA participants $(n=11)$ from the same age range (40-75 years). Scans of 7 hand OA participants and 1 control were excluded due to excessive movement artefact. Seed-based correlation analyses were performed using the CONN toolbox ${ }^{2}$ to evaluate differences in functional connectivity of networks involving the ACC, insular cortices and thalami between patients and controls, and between pre- and post-treatment states.

Results: Relative to non-OA controls, hand OA participants had increased functional connectivity at baseline between the ACC and the cunei, occipital poles, lateral occipital cortices and precuneus ( $p=0.00054$, FDR-corrected for multiple comparisons; MNI coordinates $x=-4, y=-86, z=+40 ; 513$ voxels) (figure 1 ). No differences in baseline functional connectivity were seen in the insular cortices or thalami. After treatment, altered functional connectivity was identified between the left thalamus and the ACC and paracingulate gyri $\left(\mathrm{p}_{\mathrm{FDR}}=0.038\right.$; $\mathrm{MNI}$ coordinates $x=+2, y=+36, z=+24 ; 138$ voxels). Pairwise comparisons between treatment cohorts suggested that this represented a reduction in connectivity with pregabalin relative to duloxetine, without reaching statistical significance $\left(p_{F D R}=0.062\right)$. No significant treatment-associated connectivity changes were evident between duloxetine and placebo, or pregabalin and placebo.

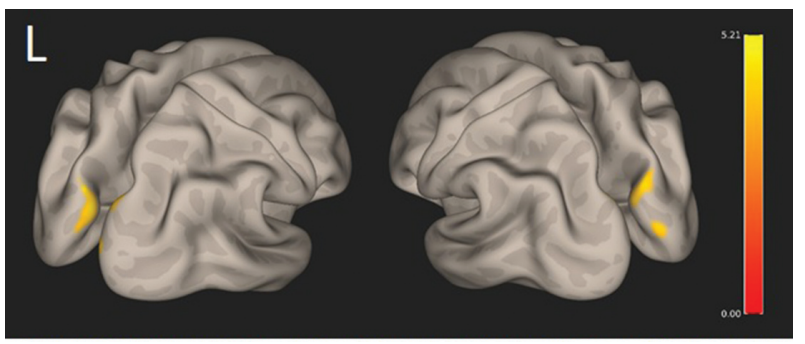

Abstract SAT0570 - Figure 1. Seed-based correlation analysis demonstrating increased baseline functional connectivity between the ACC and the cunei, occipital poles, lateral occipital cortices and precuneus in hand OA participants, relative to non-OA controls.

Conclusions: We have shown that people with painful hand OA have altered functional connectivity networks involving the ACC. In conjunction with previous findings of volumetric changes in this pain-processing brain region, this strongly supports the role of maladaptive neuroplasticity and central sensitisation in hand OA pain. Larger studies are required to better confirm if treatment with centrallyacting analgesics leads to connectivity changes in these brain regions.

\section{REFERENCES:}

[1] Russell M, Howe F, Barrick T, Sofat N. SAT0530 Central sensitisation in hand osteoarthritis: the anterior cingulate cortex is involved in pain processing. Ann Rheum Dis 2017;76:976-977.

[2] Whitfield-Gabrieli S, Nieto-Castanon A. Conn: a functional connectivity toolbox for correlated and anticorrelated brain networks. Brain Connect 2012;2(3):125-41.

Acknowledgements: We acknowledge support from the Rosetree's Trust and the NIHR Clinical Research Network.

Disclosure of Interest: None declared

DOI: 10.1136/annrheumdis-2018-eular.4107

\section{SAT0571 THE RADIOLOGICAL, CLINICAL AND FUNCTIONAL PROPERTIES OF HAND OSTEOARTHRITIS AND THEIR RELATION WITH RADIOLOGICAL FINDINGS IN A TURKISH POPULATION: TLAR-OA STUDY}

M.T. Duruöz ${ }^{1}$, D. Erdem ${ }^{1}$, T. Tuncer ${ }^{2}$, L. Altan ${ }^{3}$, F. Ayhan ${ }^{4}$, A. Bal ${ }^{5}$, L. Cerrahoglu' E. Capkin ${ }^{7}$, R. Cevik ${ }^{8}$, D. Dulgeroglu ${ }^{5}$, S. Gursoy ${ }^{9}$, S. Hizmetli ${ }^{10}$, C. Kacar ${ }^{2}$, E. Kaptanoglu ${ }^{10}$, T. Kaya ${ }^{11}$, H. Kocabaş ${ }^{12}$, K. Nas $^{13}$, S. Ozcakir ${ }^{3}$, D. Sindel ${ }^{14}$ O. Sahin ${ }^{15}$, G. Tasci Bozbas ${ }^{16}$, C. Tikiz ${ }^{6}, \mathrm{H}$. Ugurlu ${ }^{17} .^{1}$ PMR Department, Rheumatology Division, Marmara University School of Medicine, Istanbul; ${ }^{2}$ PMR Department, Rheumatology Division, Akdeniz University School of Medicine, Antalya; ${ }^{3}$ PMR Department, Uludağ University, School of Medicine, Bursa; ${ }^{4}$ PMR Department, University of Health Sciences Ankara TRH; ${ }^{5} \mathrm{PMR}$ Department, University of Health Sciences Diskapi Yildirim Beyazit TRH, Ankara; ${ }^{6} \mathrm{PMR}$ Department, Celal Bayar University School of Medicine, Manisa; ${ }^{7}$ PMR Department, Karadeniz Technical University School of Medicine, Trabzon; ${ }^{8} P M R$ Department, Dicle University School of Medicine, Diyarbakir, ${ }^{9}$ PMR Department, Gaziantep University School of Medicine, Gaziantep; ${ }^{10}$ PMR Department, Rheumatology Division, Cumhuriyet University School of Medicine, Sivas; ${ }^{11} P M R$ Department, Izmir Bozyaka TRH, Izmir, ${ }^{12}$ PMR Department, Rheumatology Division, Necmettin Erbakan University School of Medicine, Konya; ${ }^{13} \mathrm{PMR}$ Department, Sakarya University School of Medicine, Sakarya; ${ }^{14}$ PMR Department, Istanbul University School of Medicine, Istanbul; ${ }^{15}$ PMR Department, Cumhuriyet University School of Medicine, Sivas; ${ }^{16}$ PMR Department, Adnan Menderes University School of Medicine, Aydın; ${ }^{17}$ PMR Department, Necmettin Erbakan University School of Medicine, Konya, Turkey

Objectives: To evaluate the radiological, clinical and functional properties of hand osteoarthritis and to assess their relationship in a Turkish population. Methods: The subjects with hand OA recruited into the study from the multicentre national osteoarthritis cohort by the Turkish League Against Rheumatology (TLAR-OA). The demographic characteristics, body mass index (BMI), smoking finger ratio,(2nd to 4th finger lenght), grip strengths with Jamar dynamometer, hand pain duration (month), hand pain severity (visual analogue scale; 0$100 \mathrm{~mm}$ ) were evaluated. The radiological stage of hand OA was assessed according to the Kellgren Lawrence OA staging. The hightest stage among the joints involvement was noted as the OA stage of the hand. The functional disability concerning to hand involvement was assessed with Duruöz Hand Index (DHI). The SPSS 24.0 statistical package was used for analysis. The descriptive analysis was performed for all parameters. Spearman's correlation coefficient was used to assess the relation between quantitative variables.

Results: A total of 364 subjects ( 330 female, 34 male) from 15 centres with mean of age 62.96 (SD: 10.22) were recruited into the study. The mean of BMI was 29.54 (SD: 4.51 ) and the percentage of smoking person was $6.3 \%$. The mean of 2 nd finger to 4 th finger ratio of the patients was 0.96 (SD: 0.05$)$. The mean of grip strengths of the right hand and left hand were respectively $15.55 \mathrm{~kg}$ (SD: 12.88) and $14.13 \mathrm{~kg}$ (SD: 11.39), which is lower than normal population. ${ }^{1}$ The median pain duration of hand was 24 months (min-max: 1-480 months). The mean VAS score of pain was 5.05 (SD: 2.33). The mean DHI score was 16.82 (SD: 15.53). The patients' radiological OA involvement stages (2,3 and 4) of right and left hands were respectively $26.1 \%$ and $23.6 \%$ (stage 2 ); $22.5 \%$ and $20.9 \%$ (stage 3 ); $13.2 \%$ and $12.4 \%$ (stage 4 ) according to the Kellgren Lawrence scoring. The radiological stages of right and left hands respectively had poor correlation with VASpain ( $r h o=0.152, p=0.010$; rho $=0.158, p=0.009$ ); low but significant correlations with Duruöz Hand Index ( $r h o=0.257, p<0.0001$; rho $=0.267, p<0.0001$ ) and low but significant correlations with the duration of pain (rho $=0.231, p<0.0001$; $\mathrm{rho}=0.181, \mathrm{p}=0.003$ ). There were no significant correlations between the radiological stage of hand $O A$ and $B M I$, finger ratio, grip strength ( $p>0.05$ ).

Conclusions: Although the subjects with hand OA had pain, low grip strenght and hand disability; the radiological findings had meaningful relations with only functional involvement and pain duration in our population.

\section{REFERENCE:}

[1] Massy-Westropp N, et al. Hand grip strenght: age and gender stratified normative data in a population-based study. BMC Res Notes 2011;4:127

Disclosure of Interest: M. T. Duruöz Grant/research support from: ABVIE, Consultant for: NOVARTIS, Speakers bureau: ABDI IBRAHIM, D. Erdem: None declared, T. Tuncer: None declared, L. Altan: None declared, F. Ayhan: None declared, A. Bal: None declared, L. Cerrahoglu: None declared, E. Capkin: None declared, R. Cevik: None declared, D. Dulgeroglu: None declared, S. Gursoy: None declared, S. Hizmetli: None declared, C. Kacar: None declared, E. Kaptanoglu: None declared, T. Kaya: None declared, H. Kocabaş: None declared, K. Nas: None declared, S. Ozcakir: None declared, D. Sindel: None declared, O. Sahin: None declared, G. Tasci Bozbas: None declared, C. Tikiz: None declared, H. Ugurlu: None declared

DOI: 10.1136/annrheumdis-2018-eular.6546 